\title{
OBSERVATIONS OF PLASMA UPFLOW IN A WARM LOOP WITH HINODE/EIS
}

\author{
Durgesh Tripathi ${ }^{1}$, Helen E. Mason ${ }^{2}$, Giulio Del Zanna ${ }^{2}$, and Steven Bradshaw ${ }^{3}$ \\ ${ }^{1}$ Inter-University Centre for Astronomy and Astrophysics, Post Bag 4, Ganeshkhind, Pune 411 007, India \\ ${ }^{2}$ Department of Applied Mathematics and Theoretical Physics, University of Cambridge, Wilberforce Road, Cambridge CB3 0WA, UK \\ ${ }^{3}$ Department of Physics and Astronomy, Rice University, Houston, TX 77005, USA \\ Received 2012 May 9; accepted 2012 June 14; published 2012 July 2
}

\begin{abstract}
A complete understanding of Doppler shift in active region loops can help probe the basic physical mechanism involved into the heating of those loops. Here, we present observations of upflows in coronal loops detected in a range of temperatures $(\log T=5.8-6.2)$. The loop was not discernible above these temperatures. The speed of upflow was strongest at the footpoint and decreased with height. The upflow speed at the footpoint was about $20 \mathrm{~km} \mathrm{~s}^{-1}$ in Fe VIII, which decreased with temperature, being about $13 \mathrm{~km} \mathrm{~s}^{-1}$ in Fe X, about $8 \mathrm{~km} \mathrm{~s}^{-1}$ in Fe XII, and about $4 \mathrm{~km} \mathrm{~s}^{-1}$ in Fe XIII. To the best of our knowledge, this is the first observation providing evidence of upflow of plasma in coronal loop structures at these temperatures. We interpret these observations as evidence of chromospheric evaporation in quasi-static coronal loops.
\end{abstract}

Key words: Sun: atmosphere - Sun: corona - Sun: transition region - Sun: UV radiation

Online-only material: color figures

\section{INTRODUCTION}

The problem of heating and maintaining the structures in the upper solar atmosphere, such as in the transition region and corona, has been considered to be one of the most challenging issues in astrophysics. There have been tremendous developments in observations and theory in the past few decades. However, a definitive solution remains elusive. See Klimchuk (2006) for a review.

The discovery that loop structures are one of the basic building blocks of the solar corona has simplified the problem a great deal to that of understanding the heating and maintenance of individual loops. This is basically because the inherent characteristics of the corona are such that there is no cross-field conduction due to very high electric conductivity.

Recent observations have revealed that active regions are comprised of different types of loop structures: warm loops (which seem to have a temperature around $1 \mathrm{MK}$ ), fan loops (seen emanating from the umbral and penumbral regions at temperatures slightly lower than $1 \mathrm{MK}$ ), and hot core loops (seen in the core of active regions at temperatures around $2-3 \mathrm{MK})$. The heating and maintenance of fan loops and hot core loops are currently a matter of hot debate. For hot core loops, there is evidence for both steady (see, e.g., Warren et al. 2008, 2010; Brooks \& Warren 2009; Tripathi et al. 2010a; Winebarger et al. 2011) as well as impulsive heating (Tripathi et al. 2010b, 2011, 2012; Viall \& Klimchuk 2012). However, the observed characteristics of warm loops such as density, filling factors, and temperature distribution appear to be consistent with those derived from an impulsive heating model (see, e.g., Warren et al. 2003; Tripathi et al. 2009; Ugarte-Urra et al. 2009; Klimchuk 2009, and references therein).

The Doppler shift measurements in previously reported observations of warm loops show predominantly redshifted emission along the loop structures, at around 1 MK (see e.g., Del Zanna 2008; Tripathi et al. 2009). At lower temperatures however, the redshifts are localized toward the footpoints and stronger in magnitude. These downflows (redshifts) are interpreted as plasma radiatively cooling and condensing in the loops (see, e.g., Bradshaw 2008; Bradshaw \& Cargill 2010). However, the question remains with regard to how this plasma rises up into the loops at first place.

The impulsive heating scenario predicts that the plasma flows up into these loops via the mechanism of chromospheric evaporation, which occurs at relatively higher temperatures. Patsourakos \& Klimchuk (2006) predict that those high-velocity upflows will be clearly seen in Fe XVII lines, or other lines forming at similar temperatures. However, so far there has been no direct detection of these upflows. Such upflows could also be attributed to Type II spicules (see, e.g., De Pontieu et al. 2011).

Here, we present the first observations of a warm loop that shows clear upflows of the plasma at low temperatures. These observations are taken with the Extreme-ultraviolet Imaging Spectrometer (EIS; Culhane et al. 2007) on board Hinode. The Letter is structured as follows. In Section 2, we describe the observations followed by data analysis and results in Section 3 . We summarize and conclude in Section 4.

\section{OBSERVATIONS}

The EIS (Culhane et al. 2007) on board Hinode observed an active region, AR 11131, on 2010 December 11 using the study sequence which we designed (“GDZ_300x384_S2S3_35”). This sequence uses the $2^{\prime \prime}$ slit with an exposure time of $35 \mathrm{~s}$. This study sequence is designed to be a sparse raster with a step size of $3^{\prime \prime}$. The EIS raster used in this analysis started at 01:55:26 UT and was completed at 02:56 UT. We have applied standard processing software provided in solarsoft to the EIS observations.

The left image in Figure 1 displays a part of the Sun's disk recorded by the $171 \AA$ passband of Atmospheric Imaging Assembly (AIA) on board the Solar Dynamics Observatory (SDO). The emission in the $171 \AA$ passband peaks at a temperature of $\log T=6.0$, similar to the Fe $\mathrm{x} 184 \AA$ line observed by the EIS. However, depending on the individual structures being observed on the Sun, different channels on AIA may respond differently (see, e.g., O’Dwyer et al. 2010; Del Zanna et al. 2011). The overplotted bigger box shows the area which was rastered by EIS, for which a spectral image built in the Fe x $184 \AA$ is shown in the middle panel. The smaller box in the left panel 

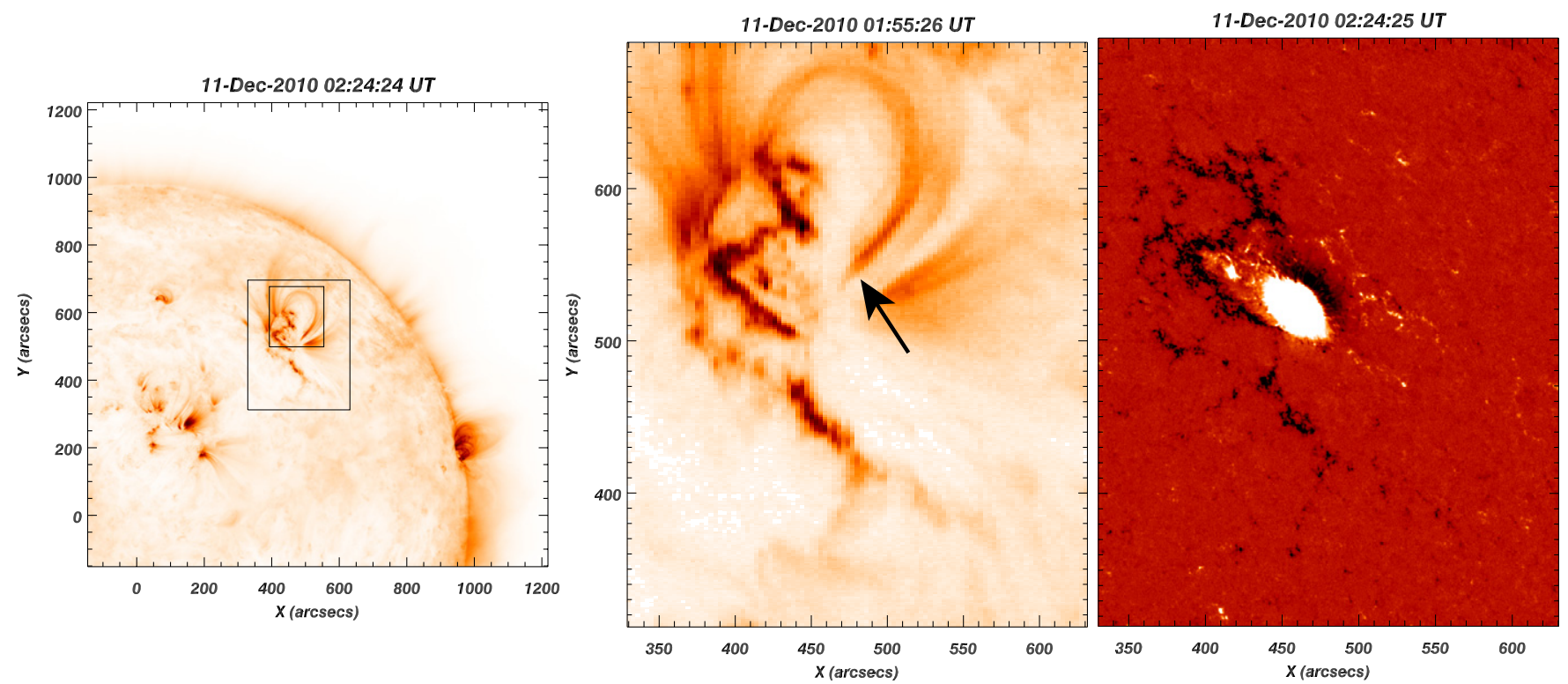

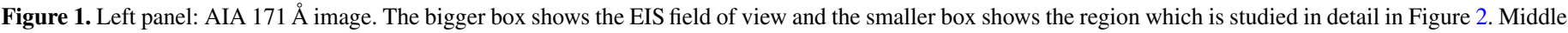

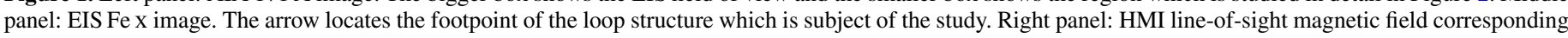
to EIS FOV. The magnetic field is scaled between -300 and $300 \mathrm{G}$.

(A color version of this figure is available in the online journal.)

represents the region which was further studied in detail by AIA. The right panel shows the line-of-sight magnetogram obtained by the Helioseismic and Magnetic Imager (HMI) aboard $S D O$ for the region corresponding to the EIS raster. In the magnetogram, white regions represent the positive magnetic field and dark represent the negative magnetic field regions. AIA and HMI images are taken when the EIS slit would have been approximately at the middle of the raster. It is worthwhile to note that we have co-aligned EIS and AIA images just by eye. We believe that the co-alignment is accurate to a level of about 7-8 arcsec. The loop structure, on which this Letter is focused, is located by an arrow. These images clearly show that the western footpoint of the loop is rooted in the sunspot seen in the right panel. The eastern moss region corresponding to negative polarity regions is more dispersed. It is worthwhile to note that the moss regions are seen only toward the negative polarity region as noted earlier by Tripathi et al. (2008).

Since we are interested in an understanding of quiescent coronal loops, we analyzed the AIA data for about $5 \mathrm{hr}$ (a couple of hours before and after the observations) to make sure that the loop being analyzed here was quasi-static and that the active region did not show any flaring and/or micro-flaring activity. Figure 2 displays AIA images recorded in the $171 \AA$ band corresponding to the smaller box shown in the left panel of Figure 1. It is clear from these images that there are no major changes in the overall structures of the loops. However, some small variations in the intensity toward the footpoint region can be seen. The results presented here relate to quiescent (1 MK) coronal loops seen with Transition Region and Coronal Explorer or $S D O /$ AIA at $171 \AA$.

\section{DATA ANALYSIS AND RESULTS}

Figure 3 shows intensity maps derived in $\mathrm{Mg}$ v $276 \AA$, Mg VII $278 \AA, \operatorname{Mg}$ VII $280 \AA$, Fe VIII $186 \AA$, Fe x $184 \AA$, Fe XI $188 \AA$, Fe XII $195 \AA$, Fe XIII $202 \AA$, and Fe XV $284 \AA$. As can be seen from these images, the loop structures (shown with arrows in middle left panel) are clearly discernible in Mg viI $278 \AA$,
Si VII $275 \AA$, and Fe IX $188 \AA$. Only a tiny portion of the loop at the footpoint, labeled with an arrow in the top row middle panel, is seen in the $\mathrm{Mg} v$ line. The footpoint of the loop seen in the $\mathrm{MgV}$ image is thinner than the same part of the loop seen in other lines such as Si VII and Fe IX. For the higher temperature lines such as Fe $\mathrm{x}$ the contrast between loop and the background/foreground fuzzy emission has decreased and it becomes almost impossible to discern the loop in Fe XIII. In other words, the corona becomes fuzzier with increasing temperature as was shown with Hinode/EIS data by Tripathi et al. (2009). It is probably worth emphasizing that the intensities in the loop structures are just about 10\%-20\% higher than the background/foreground coronal intensities (see, e.g., Del Zanna \& Mason 2003; Viall \& Klimchuk 2012). It is important to note that the intensities in the background/foreground regions are higher in the images obtained in lines like Fe XII and Fe XIII, but decreases in Fe Xv. These images also suggest that there are loops at different temperatures intermingled together which can only be distinguished uniquely by spectroscopic observations.

Comparing the structures in AIA images (Figure 2, spatial resolution $\approx 1$ arcsec) and with that in EIS images (Figure 3, spatial resolution $\approx 3-4$ arcsec) it is clear that the loop structure which appears to be an individual structure in EIS images, are comprised of several different flux tubes as seen with AIA. A comparison between AIA, EIS, and HMI observations reveals that the western footpoints of the loop structures under consideration are rooted in the sunspot (leading polarity) and the eastern footpoints are rooted in more fragmented following polarity magnetic field regions.

The footpoint of the loop, shown by an arrow labeled " $F$ " in the left panel in the middle row of the Figure 3, is brightest. With increasing height, the emission from the loop gets fainter. A closer look at the footpoint region suggests that there are at least three different loops, marked with three arrows higher up, emanating from either the same location or close by. The footpoint regions of these three loops seem to coincide along the line of sight which could possibly lead to brighter emission near the footpoints. 

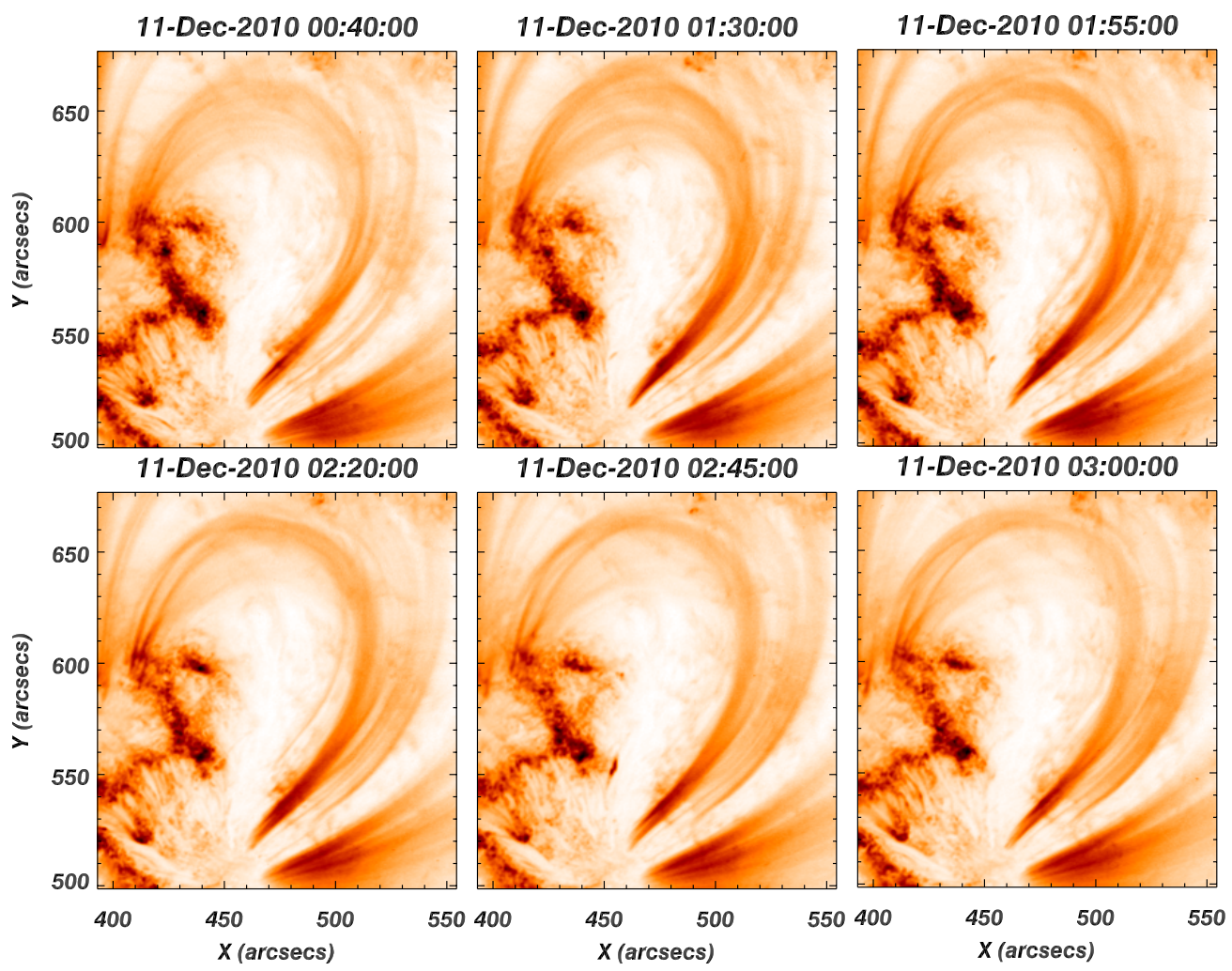

Figure 2. AIA $171 \AA$ images corresponding to the small box shown in Figure 1 . The images are shown before, during, and after the EIS raster. (A color version of this figure is available in the online journal.)

The two right panels in the top row in Figure 3 show intensity maps obtained using two lines of Mg vII, namely, $278 \AA$ and $280 \AA$. The loop is clearly visible in Mg viI $278 \AA$, but only a part of the loop is visible in Mg VII $280 \AA$. We note that the ratio of $\mathrm{Mg}$ VII $280 \AA$ to $\mathrm{Mg}$ VII $278 \AA$ is sensitive to electron number density. This indicates that the electron density is very low toward the upper part of the loop.

Deriving Doppler shifts from EIS observations is a non-trivial as well as a non-unique process (see, e.g., Kamio et al. 2010; Young et al. 2012; Tripathi et al. 2012). Most important of all is to remove the orbital variation of the spacecraft and to determine the reference wavelength. Doppler shifts can then be derived. There are a few different ways to derive Doppler shifts from EIS data (see, e.g., Kamio et al. 2010; Dadashi et al. 2011; Young et al. 2012). In this Letter, we have used the method proposed by Young et al. (2012). This is based on deriving velocities (using Fe vIII as a reference) in the quiet-Sun region of each slit position in the raster. Following this method, we have used the Fe VIII 186.6 $\AA$ line in the quiet Sun to derive Doppler shifts for other lines. We have chosen the bottom 30 rows in the raster to be the quiet Sun. The only difference between our method and that of Young et al. (2012) is that they calibrated the quiet Sun using the Solar Ultraviolet Measurement of Emitted Radiation measurements from Peter \& Judge (1999), whereas we choose the Fe VIII line to have zero Doppler shift, consistent with the new values of Dadashi et al. (2011). For more details on this method, the reader is referred to Young et al. (2012).

Figure 4 shows the velocity maps derived for Fe VIII, Fe X, Fe XII, and Fe XIII. The overall velocity structure in the active region appears to be the similar to those published earlier (see, e.g., Doschek et al. 2008; Del Zanna 2008; Tripathi et al. 2009), with a weakly redshifted active region core and strongly blueshifted low intensity regions. The core of the active region show strong redshifts for the Fe VIII line. This is because the Fe vIII $186 \AA$ line is blended in its redwing with a Ca XIV line which forms at $\log T=6.4$. Since the core of the active region is characterized by high-temperature emission, the Doppler shift for Fe VIII is overestimated. The loop structures marked by arrows in Figure 3 are blueshifted, unlike the earlier reported observations of strongly redshifted warm loops. The blueshifted loops are marked by arrows in Figure 4. The loop which is seen along its entire length is blueshifted all along the loop in the $\mathrm{Fe}$ vIII line and both the footpoints are visible. For other spectral lines shown in Figure 4, only one footpoint of the loop is visible and is blueshifted.

To understand the correlation between the loop intensity and its velocity structure better, we plot intensity and velocity profiles along three different horizontal cuts shown in the top left panel of Figure 5 as "Region 1," "Region 2," and "Region 3." The remaining three panels show the intensity and velocity profiles for three regions. These intensities are plotted for the Fe VIII line and the corresponding velocity profiles are plotted for Fe VIII, Fe X, Fe XII, and Fe XIII. In the plots, the strong spikes (intensity enhancement) in the intensity profile correspond to the loops marked in Figure 3. As anticipated, the loop intensity decreases with height. Similar characteristics are seen in the velocity profile. The loop is strongly blueshifted at the footpoint with a speed of about $20 \mathrm{~km} \mathrm{~s}^{-1}$ in Fe VIII. The velocity of the upflow at the footpoint decreases with temperature, being about $13 \mathrm{~km} \mathrm{~s}^{-1}$ in Fe X, about $8 \mathrm{~km} \mathrm{~s}^{-1}$ in Fe XII, and about $4 \mathrm{~km} \mathrm{~s}^{-1}$ in Fe XIII. In addition, the upflow velocity also decreases with the height of the loop. We note that these speeds are lower limits of the actual speed of the plasma, since we are measuring the line-of-sight component of the speeds. Similar, though less pronounced, characteristics can be seen for other loops adjacent to the strongly emitting loop structure. 

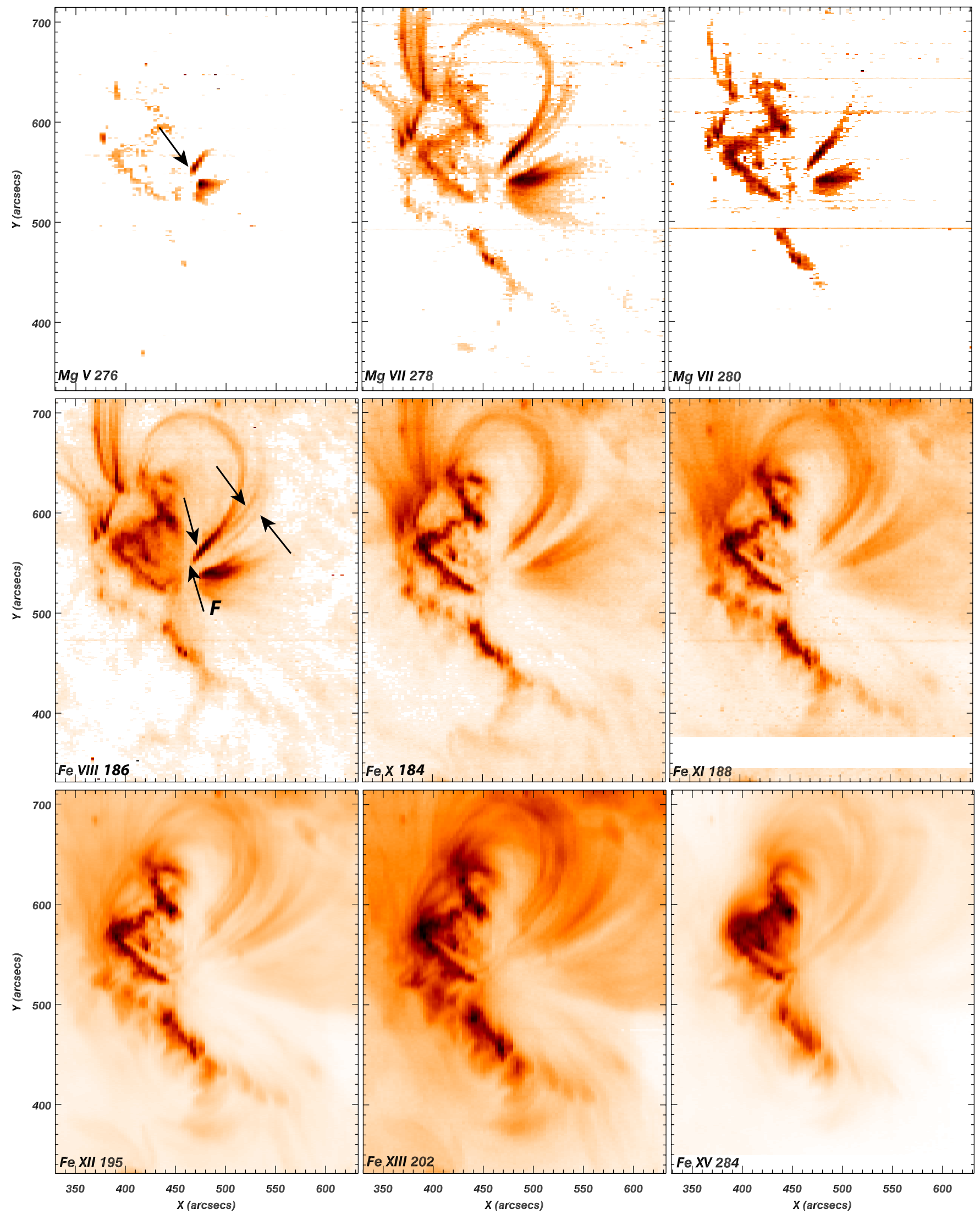

Figure 3. Monochromatic intensity maps obtained using EIS spectra. The arrow shown in the top left panel shows the footpoint of the loop seen in Mg $\mathrm{v}$ which is also shown by an arrow in top right panel labeled "F." The three arrows shown in the top right panel locate three distinguished loops.

(A color version of this figure is available in the online journal.) 

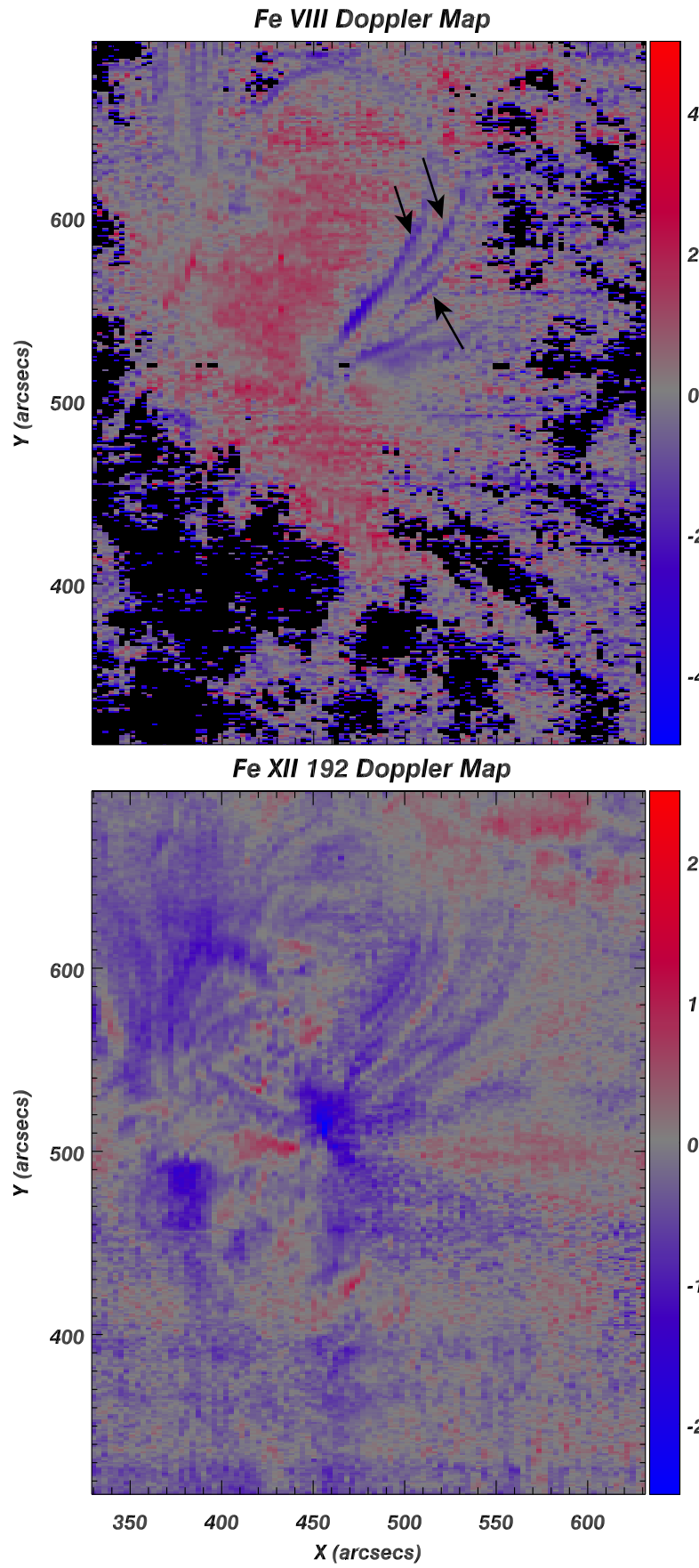

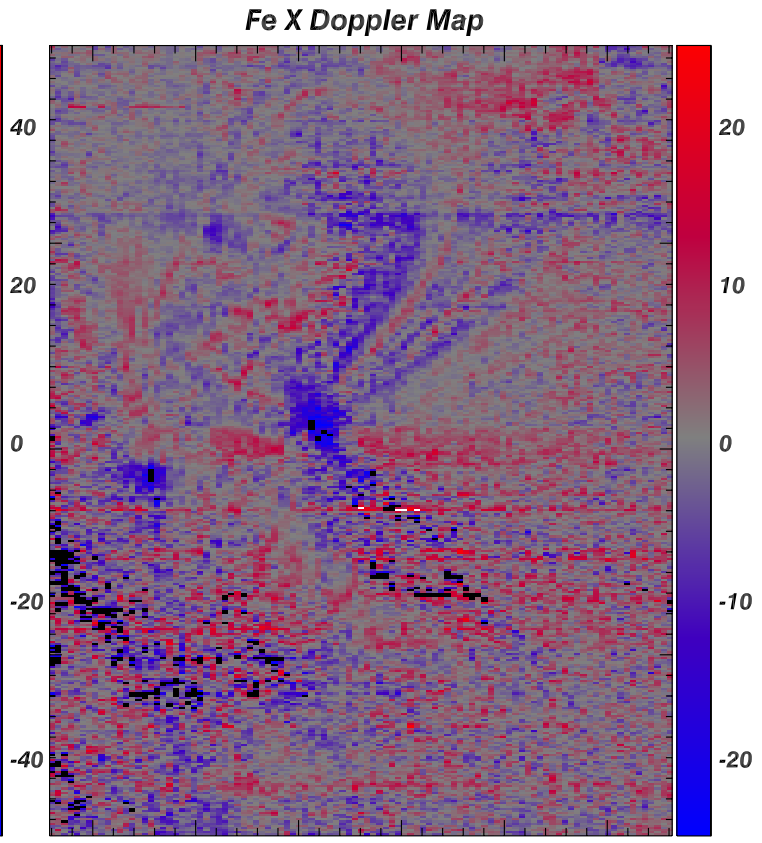

Fe XIII 202 Doppler Map

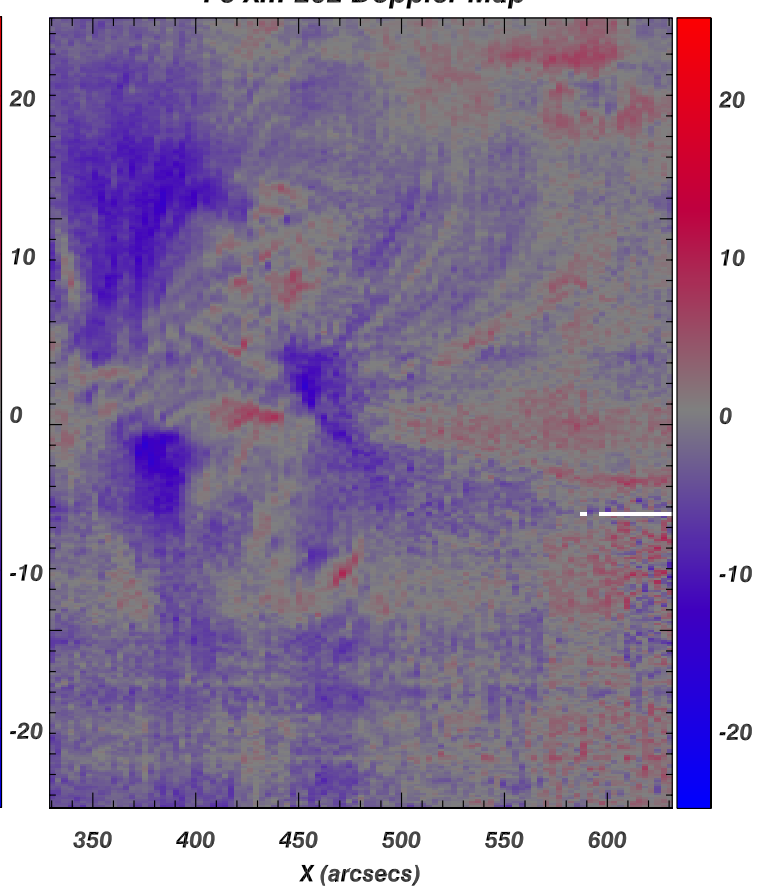

Figure 4. Doppler maps for Fe vIII, Fe X, Fe XII, and Fe XIII.

(A color version of this figure is available in the online journal.)

The peak of the intensity and velocity profiles for the loops do not coincide with each other. The peak of the velocity seems to be shifted $3^{\prime \prime}$ eastward with respect to the peak in intensity. This is most likely due to the tilted point-spread function of EIS as was pointed out by Young et al. (2012). Young et al. (2012) found that strongly redshifted or blueshifted regions are often seen with some offset from the regions with the highest intensity point. Investigating many different data sets, they concluded that this could be attributed to a tilted point-spread function of the instrument. In one observation presented in Young et al. (2012) it was found that there was a difference of $3^{\prime \prime}$ between the intensity peak and velocity peak in the $Y$-direction. In these circumstances, Young et al. (2012, pp. 16-17) state, "the simplest way of deriving velocity shifts for features with steep intensity gradients is either to study only those pixels at the location of the intensity peaks in the solar $Y$-direction, or to perform averaging in the $Y$-direction over a region symmetrically distributed around the intensity peak." In the present observations, even if we average the velocity over the width of the loop in $X$-direction, the blueshifted pattern remains. Therefore, we conclude that this blueshift seen along the loop is real and not due to some instrumental artifact.

\section{SUMMARY AND DISCUSSION}

We have presented an observation of the active region AR 11131 recorded on 2010 December 11 by Hinode EIS. The active region is comprised of warm loops, fan loops, and hot 

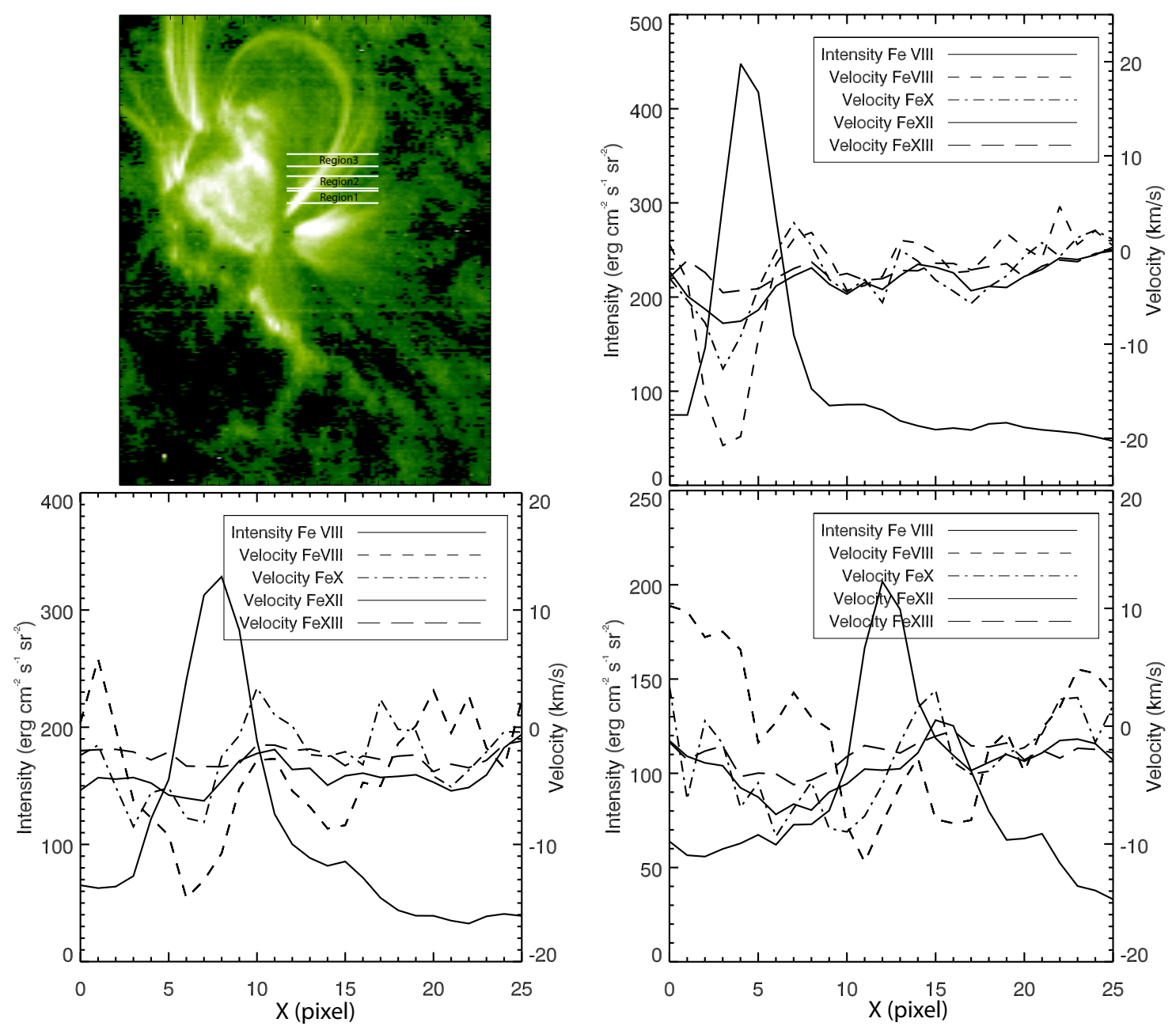

Figure 5. Correspondence between Fe VIII intensity profile and velocity profiles for Fe VIII, Fe X, Fe XII, and Fe XIII.

(A color version of this figure is available in the online journal.)

core loops among other features, such as moss, etc. The intensity and velocity structure of the active region show characteristics similar to those published earlier by, e.g., Doschek et al. (2008), Del Zanna (2008), and Tripathi et al. (2009). However, in contrast to the observations reported earlier, where the warm loops at around $1 \mathrm{MK}$ have been shown to be redshifted, the warm loops in this active region are blueshifted (see Figures 3 and 4). The footpoint of the loop is strongly blueshifted with a speed of about $20 \mathrm{~km} \mathrm{~s}^{-1}$ in Fe VIII. The upflow velocity at the footpoint decreases with increasing temperature as well as the height of the loop. To the best of our knowledge this is the first observational report of a blueshifted warm loop.

Based on the multi-stranded impulsive heating scenario, chromospheric evaporation of plasma into coronal loop structures takes place at rather very high temperatures: Patsourakos \& Klimchuk (2006) predict high-velocity upflows in spectral lines like Fe XVII. In this scenario, the heating takes place in the corona. Energy is conducted downward, leading to chromospheric evaporation into the corona. At the temperature around which lines like Fe vIII would form, most of the strands would be cooling down and therefore the loop emission is expected to be dominated by redshifted emission. So, the observations presented here do not seem to show the flow structure expected from these multi-stranded loop models. However, it is important to emphasize here that in these models plasma flow characteristics change quite significantly when the characteristics of the heating events are changed. The characteristics of the heating events are ad hoc. If the energy of the heating events is significantly higher, then it may well be that high-velocity upflows will be seen at higher temperatures and later on the total emission will be dominated by plasma condensation. However, if the energy and duration of the heating event is short, the plasma may behave differently and the model may predict upflows at significantly lower temperature, making our observations consistent with an impulsive heating scenario. However, this scenario predicts increasing upflow velocity with temperature, which is opposite to what we observe here.

Our observation is also consistent with the picture that the heating takes place at the footpoints of the loops, i.e., lower down in the atmosphere rather than higher up in the corona (see, e.g., Aschwanden et al. 2007). In this scenario too, because of heat deposition lower down, the pressure will increase, leading to plasma evaporation into the loops. If the heat deposition is lower in the atmosphere then evaporation at temperatures of about $1 \mathrm{MK}$ would result in blueshifted emission in the loops, similar to our observations. In fact this is argued to be one of the best evidences for footpoint heating (see, e.g., Aschwanden et al. 2007).

Lastly, the observations presented here could possibly be consistent with the newly proposed mechanism of heating of the solar corona by Type II spicules (De Pontieu et al. 2011). In this scenario, the chromospheric material is being pumped into the corona by these spicules and heated. 
To the best of our knowledge, these observations present the first clear case of the upflow of plasma in coronal loops and provide an important constraint on the theories of coronal heating. Clearly, more observations and theoretical work are needed before anything conclusive can be said about the heating of warm loops. Future missions such as the Interface Region Imaging Spectrometer, the Spectral Imaging of Coronal Environment on board Solar Orbiter, and the proposed Large European module for solar Ultraviolet Research (LEMUR) on board Solar-C will provide unique and definitive observations to study such problems.

We thank the referee for providing useful inputs which has improved the Letter. H.E.M. and G.D.Z. acknowledge S.T.F.C. We acknowledge useful discussions at the ISSI on Active Region Heating. Hinode is a Japanese mission developed and launched by ISAS/JAXA, collaborating with NAOJ as a domestic partner, and NASA and STFC (UK) as international partners. Scientific operation of the Hinode mission is conducted by the Hinode science team organized at ISAS/JAXA. This team mainly consists of scientists from institutes in the partner countries. Support for the post-launch operation is provided by JAXA and NAOJ (Japan), STFC (U.K.), NASA, ESA, and NSC (Norway).

\section{REFERENCES}

Aschwanden, M. J., Winebarger, A., Tsiklauri, D., \& Peter, H. 2007, ApJ, 659 1673

Bradshaw, S. J. 2008, A\&A, 486, L5
Bradshaw, S. J., \& Cargill, P. J. 2010, ApJ, 717, 163

Brooks, D. H., \& Warren, H. P. 2009, ApJ, 703, 10

Culhane, J. L., Harra, L. K., James, A. M., et al. 2007, Sol. Phys., 243, 19

Dadashi, N., Teriaca, L., \& Solanki, S. K. 2011, A\&A, 534, 90

Del Zanna, G. 2008, A\&A, 481, L49

Del Zanna, G., \& Mason, H. E. 2003, A\&A, 406, 1089

Del Zanna, G., O’Dwyer, B., \& Mason, H. E. 2011, A\&A, 535, L46

De Pontieu, B., McIntosh, S. W., Carlsson, M., et al. 2011, Science, 331, 55

Doschek, G. A., Warren, H. P., Mariska, J. T., et al. 2008, ApJ, 686, 1362

Kamio, S., Hara, H., Watanabe, T., Fredvik, T., \& Hansteen, V. H. 2010, Sol. Phys., 266, 209

Klimchuk, J. A. 2006, Sol. Phys., 234, 41

Klimchuk, J. A. 2009, in ASP Conf. Ser. 415, The Second Hinode Science Meeting: Beyond Discovery-Toward Understanding, ed. B. Lites et al. (San Francisco, CA: ASP), 221

O’Dwyer, B., Del Zanna, G., Mason, H. E., Weber, M. A., \& Tripathi, D. 2010, A\&A, 521, 21

Patsourakos, S., \& Klimchuk, J. A. 2006, ApJ, 647, 1452

Peter, H., \& Judge, P. G. 1999, ApJ, 522, 1148

Tripathi, D., Klimchuk, J. A., \& Mason, H. E. 2011, ApJ, 740, 111

Tripathi, D., Mason, H. E., Del Zanna, G., \& Young, P. R. 2010a, A\&A, 518, 42

Tripathi, D., Mason, H. E., Dwivedi, B. N., del Zanna, G., \& Young, P. R. 2009, ApJ, 694, 1256

Tripathi, D., Mason, H. E., \& Klimchuk, J. A. 2010b, ApJ, 723, 713

Tripathi, D., Mason, H. E., \& Klimchuk, J. A. 2012, ApJ, 753, 37

Tripathi, D., Mason, H. E., Young, P. R., \& Del Zanna, G. 2008, A\&A, 481, L53

Ugarte-Urra, I., Warren, H. P., \& Brooks, D. H. 2009, ApJ, 695, 642

Viall, N. M., \& Klimchuk, J. A. 2012, ApJ, 753, 35

Warren, H. P., Winebarger, A. R., \& Brooks, D. H. 2010, ApJ, 711, 228

Warren, H. P., Winebarger, A. R., \& Mariska, J. T. 2003, ApJ, 593, 1174

Warren, H. P., Winebarger, A. R., Mariska, J. T., et al. 2008, ApJ, 677, 1395

Winebarger, A. R., Schmelz, J. T., Warren, H. P., Saar, S. H., \& Kashyap, V. L. 2011, ApJ, 740, 2

Young, P. R., O’Dwyer, B., \& Mason, H. E. 2012, ApJ, 744, 14 